Results 156 (105 women and 51 men) healthy, immunocompetent, HSV-2 positive participants with a history of $1-9$ recurrences per year prior to trial entry, or previous suppressive therapy, were randomised by seven US sites between May 2010 and October 2010. 147 completed the trial. Overall, about 9000 swabs for HSV PCR were collected and assayed for HSV DNA by a sensitive and accurate assay that can detect $>150$ copies $/ \mathrm{ml}$. The first results of these assessments will be presented.

Conclusion The trial will provide insight into the antiviral activity of the novel agent AIC316 for genital HSV infections. This trial design presents a robust and efficient method for evaluating antiviral activity of candidate agents for mucocutaneous HSV infections. These initial efficacy and safety results will lead to selecting the dose for further trials with AIC316.

\section{3-S5.02 FREQUENT BREAKTHROUGH GENITAL HSV-2 SHEDDING ON STANDARD AND HIGH DOSE VALACYCLOVIR}

doi:10.1136/sextrans-2011-050109.128

${ }^{1} \mathrm{C}$ Johnston, ${ }^{2} \mathrm{M}$ Saracino, ${ }^{3} \mathrm{~S}$ Kuntz, ${ }^{1} \mathrm{~A}$ Magaret, ${ }^{1} \mathrm{~J}$ T Schiffer, ${ }^{2} \mathrm{~S}$ Selke, ${ }^{1}$ Meeili Huang, ${ }^{4} \mathrm{~L}$ Corey, ${ }^{1} \mathrm{~A}$ Wald. ${ }^{1}$ University of Washington UW, Fred Hutchinson Cancer Research Center, Seattle, USA; ${ }^{2}$ University of Washington, USA; ${ }^{3}$ Laboratory Medicine, USA; ${ }^{4}$ Fred Hutchinson Cancer Research Center FHCRC, University of Washington UW, USA

Background Short, rapidly cleared, subclinical shedding episodes are the predominant form of HSV-2 reactivation in the genital tract. Valacyclovir $500 \mathrm{mg}$ once daily (SD-VAL) reduces the risk of sexual transmission of herpes simplex virus type 2 (HSV-2) by only $48 \%$. We hypothesised that short HSV-2 shedding episodes occur frequently on SD-VAL and that high dose (HD)-VAL could suppress such episodes of genital HSV-2 shedding.

Methods A randomised open-label crossover study using valacyclovir $500 \mathrm{mg}$ daily (SD-VAL) vs valacyclovir $1 \mathrm{gm}$ three times daily (HD-VAL) was conducted in HSV-2 seropositive, HIV seronegative persons with four or more genital herpes recurrences per year or laboratory confirmed primary genital HSV-2 infection in the previous 6 months. Each study arm lasted for 5 weeks, separated by 1 week wash out. Participants obtained genital swabs four times daily, which were assayed for HSV by quantitative PCR. The primary outcome was frequency of genital HSV shedding on each study arm; secondary outcomes included number and duration of HSV-2 shedding episodes and quantity of virus detected.

Results Forty-three participants collected 9981 genital swabs during the study period. 292 (5.8\%) of 5008 swabs had HSV detected during SD-VAL, compared to 164 (3.3\%) of 4973 on HD-VAL (IRR=0.52, $95 \% \mathrm{CI}=0.43 \%$ to $0.63 \%, \mathrm{p}<0.001)$. Episodes were shorter on $\mathrm{HD}$ VAL (median $7 \mathrm{~h}$, compared to $10 \mathrm{~h}$ on SD-VAL, $\mathrm{p}=0.03$ ) and the median maximum copy number was lower on HD-VAL (3.0 log10 copy $/ \mathrm{ml}$ vs $2.5 \log 10$ copies $/ \mathrm{ml}, \mathrm{p}=0.001)$. However, the annual episode rate was the same regardless of dose; there were 55 shedding episodes over 3.89 person-years of follow-up during SD-VAL (14.1 episodes/year) and 65 episodes over 3.93 person-years during HDVAL (16.5 episodes/year, $\mathrm{p}=0.34$ ).

Conclusion Short bursts of genital HSV-2 reactivation persist during SD-VAL and HD-VAL. Compared to SD-VAL, HD-VAL decreased shedding frequency and episode duration but did not alter episode rate. These data may explain why risk of HSV transmission and HSV-specific genital inflammation persist even in the presence of antiviral therapy. More potent therapies are needed to completely suppress HSV-2 reactivation

ClinicalTrials.gov number NCT00362297.

\section{3-S5.03 HIGH-DOSE VALACYCLOVIR DECREASES PLASMA HIV-1 LEVELS MORE THAN STANDARD DOSE ACYCLOVIR IN HIV-1, HSV-2 POSITIVE PERSONS: A RANDOMISED, CROSSOVER TRIAL}

doi:10.1136/sextrans-2011-050109.129

T Perti, J Baeten, C Johnston, K Diem, N Ochbamichael, Meei-Li Huang, S Selke, A Magaret, L Corey, A Wald. University of Washington, Seattle, USA

Background Standard doses of HSV suppressive therapy reduce plasma HIV-1 levels (0.25-0.5 log10 copies/mL) among HIV-1/HSV2 co-infected persons, and modestly slow disease progression. Putative mechanisms for this effect include direct inhibition of HIV1 by acyclovir or indirect reduction by decreasing HSV-associated inflammation. We hypothesised that higher-dose anti-HSV therapy would result in greater reduction in plasma HIV-1 RNA, and that the effect would be mediated by greater suppression of HSV shedding.

Methods 34 participants with HIV-1 and HSV-2 who were not on antiretroviral therapy were enrolled into a randomised, open-label cross-over trial with valacyclovir $1000 \mathrm{mg}$ twice daily or acyclovir $400 \mathrm{mg}$ twice daily for 12 weeks. After a 2 week wash-out, they were crossed over to the alternate treatment arm for 12 weeks. HSV PCR was performed on self-collected genital swabs obtained daily during the first 4 weeks of each treatment period. Plasma HIV-1 RNA was measured weekly throughout the study.

Results Among the 26 participants who completed both arms of the study, the mean age was 44; 21 were men. At entry, mean CD4 count was 525 cells/mm3 (range, 242-1055) and mean plasma HIV1 RNA $3.9 \log 10$ copies/ml (range 1.2-5.5). The mean plasma HIV RNA was $3.86 \log 10$ copies/ml during acyclovir administration compared with $3.57 \log 10$ copies/ml on valacyclovir; a $0.29 \log 10$ copies $/ \mathrm{ml}$ reduction $(\mathrm{p}=0.002)$. One week after initiation of valacyclovir, plasma HIV RNA decreased by a mean of $0.40 \log 10$ copies/ $\mathrm{ml}$. Valacyclovir reduced HIV-1 RNA by $\geq 0.25 \log 10$ copies $/ \mathrm{ml}$ in 14 (54\%) participants, compared with $3(12 \%)$ on acyclovir. Neither the HSV shedding rate $(8.92 \%$ vs $8.98 \%$ of days, $\mathrm{p}=0.94)$, nor the genital lesion rate $(4.3 \%$ vs $1.1 \% ; p=0.18)$ differed on acyclovir vs valacyclovir.

Conclusions High-dose valacyclovir reduces plasma HIV-1 RNA levels more effectively than standard dose acyclovir in HIV-1, HSV-2 seropositive persons not receiving antiretroviral therapy. High dose valacyclovir does not provide more potent suppression of HSV reactivation in HIV-1 infected persons than acyclovir, suggesting that the effect of valacyclovir on HIV-1 RNA may not be mediated via HSV suppression.

\section{3-S5.04 THE POST-TRIAL EFFECT OF PERIODIC PRESUMPTIVE TREATMENT FOR VAGINAL INFECTIONS ON THE INCIDENCE OF BACTERIAL VAGINOSIS AND LACTOBACILLUS COLONISATION}

doi:10.1136/sextrans-2011-050109.130

${ }^{1} \mathrm{~J}$ Balkus, ${ }^{2} \mathrm{~W}$ Jaoko, ${ }^{3} \mathrm{~K}$ Mandaliya, ${ }^{1} \mathrm{~B}$ Richardson, ${ }^{1} \mathrm{~L}$ Masese, ${ }^{2} \mathrm{R}$ Gitau, ${ }^{2} \mathrm{~J}$ Kiarie, ${ }^{4} \mathrm{~J}$ Marrazzo, ${ }^{4} \mathrm{C}$ Farquhar, ${ }^{4} \mathrm{R}$ S McClelland. ${ }^{1}$ University of Washington, Seattle, USA; ${ }^{2}$ University of Nairobi, Kenya; ${ }^{3}$ Coast Provincial General Hospital, USA; ${ }^{4}$ University of Washington, USA

Background Bacterial vaginosis (BV) is a highly prevalent infection that frequently recurs following standard treatment. In a randomised controlled trial (RCT) of oral periodic presumptive treatment (PPT) to reduce vaginal infections among Kenyan women, we observed a decrease in BV and an increase in Lactobacillus colonisation among women randomised to receive $2 \mathrm{~g}$ metronidazole $+150 \mathrm{mg}$ fluconazole monthly for 12 months. After the trial, 
women were invited to continue follow-up in an open cohort study. These post-trial data were analysed to test the hypothesis that the treatment effect would persist in the absence of PPT.

Methods Data were obtained from women who completed all 12 RCT visits and attended $\geq 1$ cohort study visit within 120 days of their final RCT visit. We used Andersen-Gill proportional hazards models to estimate the post-trial effect of the intervention vs placebo on the incidence of BV by Gram stain (Nugent score $\geq 7$ ) and Lactobacillus species by culture on Rogosa agar.

Results The RCT enrolled 310 subjects ( 155 per arm), of whom 165 (83 active and 82 placebo) were included in this analysis. Included subjects were slightly older (median (IOR): 33 years (29-39) vs 30 years $(26-35) ; \mathrm{p}<0.001)$ and reported a longer duration of sex work (median (IOR): 6 years $(2-11)$ vs 3 years $(1-6) ; p<0.001$ ) compared to those excluded. At the final RCT visit, which represented the baseline visit for this analysis, demographic and behavioural characteristics were similar by arm. The prevalence of BV at the final RCT visit was $16 \%$ in the active arm and $43 \%$ in the placebo arm $(p<0.001)$. The post-trial incidence of BV was $260 / 100$ person-years (p-yrs) in the active arm vs $358 / 100$ p-yrs in the placebo arm (HR=0.76; $95 \% \mathrm{CI}: 0.51 \%$ to $1.12 \%)$. The prevalence of Lactobacillus colonisation at the final RCT visit was $17 \%$ in the active arm and $18 \%$ in the placebo arm $(p=0.81)$. The post-trial incidence of Lactobacillus colonisation was 180/100 p-yrs in the active arm vs $127 / 100$ p-yrs in the placebo arm ( $\mathrm{HR}=1.42 ; 95 \% \mathrm{CI}$ : $0.85 \%$ to $2.71 \%$ ).

Conclusions Despite a decrease in BV and an increase in Lactobacillus colonisation during the RCT, the effect of PPT was not sustained during the 120 days following cessation of the intervention. New interventions that reduce BV recurrence and promote long-term Lactobacillus colonisation without the need for ongoing PPT or suppressive therapy are needed.

\section{3-S5.05 RPR TITRE VARIATION FOLLOWING EARLY SYPHILIS THERAPY: A POTENTIAL CONFOUNDER OF TREATMENT OUTCOME ASSESSMENT}

doi:10.1136/sextrans-2011-050109.131

${ }^{1} \mathrm{~K}$ Holman, ${ }^{2} \mathrm{M}$ Wolff, ${ }^{3} \mathrm{~A}$ Seña, ${ }^{4} \mathrm{D}$ Martin, ${ }^{3} \mathrm{~F}$ Behets, ${ }^{5} \mathrm{~K}$ Van Damme, ${ }^{3} \mathrm{P}$ Leone, ${ }^{6} \mathrm{~L}$ McNeil, ${ }^{2} \mathrm{~J}$ Winestone, ${ }^{1} \mathrm{E}$ Hook III. ${ }^{1}$ University of Alabama at Birmingham, Birmingham, USA; ${ }^{2}$ Emmes Corporation, Rockville, USA; ${ }^{3}$ University of North Carolina at Chapel Hill, Chapel Hill, USA; ${ }^{4}$ Louisiana State University, Baton Rouge, USA; ${ }^{5}$ University of North Carolina at Madagascar, Madagascar; ${ }^{6}$ Family Health International Research, USA

Objective Serologic tests for syphilis (STS) results at the time of diagnosis are the basis for evaluating response to syphilis therapy. Following treatment, however, STS titres may continue to increase for several weeks. In a recent study comparing azithromycin to penicillin or doxycycline for early syphilis treatment, patients had RPR titres measured initially, at 7 and at 14 days following treatment. We evaluated variation in RPR titres over the 14 days following therapy, hypothesising that RPR titre changes would vary with stage and initial titre.

Methods Prospectively identified HIV-seronegative participants at five North American and three Madagascar sites with primary, secondary or early latent syphilis were randomly assigned to penicillin, doxycycline (in the case of penicillin allergy) or azithromycin treatment. Blood for RPR analysis was drawn at days 0,7 , and 14 post-treatment. All RPR titres were determined simultaneously at a central laboratory. Analysis was done using SAS 9.2.

Results 465 patients had data available for at least 2 of 3 RPR measurements. Median RPR at diagnosis by stage was Primary 1:16, Secondary 1:64, Early Latent 1:32. Overall, 20\% of patients showed a titre increase of at least one dilution in the 14 days following therapy. Of this group, $88.2 \%$ demonstrated an increase of 1 dilution, while $11.8 \%$ demonstrated an increase of $\geq 2$ dilutions. The greatest proportion of titre increases following therapy was seen in patients with primary syphilis.

Conclusions Given the reliance upon changes in RPR titres for evaluating response to therapy, these changes in titre following therapy could affect whether a response is classified as treatment success/failure or serofast status. Further analyses will evaluate factors associated with increasing RPR titres following therapy, as well as the effect of these changes in titre on evaluation of response to therapy.

\section{3-S5.06 DOUBLE-BLIND RANDOMISED PLACEBO CONTROLLED TRIAL OF ORAL METRONIDAZOLE IN COMBINATION WITH EITHER VAGINAL CLINDAMYCIN OR AN OESTROGEN-CONTAINING VAGINAL PROBIOTIC FOR THE TREATMENT OF BACTERIAL VAGINOSIS}

doi:10.1136/sextrans-2011-050109.132

${ }^{1} \mathrm{C}$ Bradshaw, ${ }^{2} \mathrm{M}$ Pirotta, ${ }^{2} \mathrm{~J}$ Hocking, ${ }^{3} \mathrm{~S}$ Garland, ${ }^{2} \mathrm{D}$ de Guigand, ${ }^{4} \mathrm{G}$ Fehler, ${ }^{2} \mathrm{~A}$ Morrow, ${ }^{2} \mathrm{~S}$ Walker, ${ }^{2} \mathrm{~L}$ Vodstrcil, ${ }^{5} \mathrm{C}$ Fairley. ${ }^{1}$ Melbourne Sexual Health Centre, University of Melbourne, Melbourne, Australia; ${ }^{2}$ University of Melbourne, Australia; ${ }^{3}$ Royal women's hospital, Australia; ${ }^{4}$ Melbourne sexual health centre, Australia; ${ }^{5}$ University of Melbourne, Melbourne sexual health centre, Australia

Background To determine if addition of vaginal clindamycin or an oestrogen-containing vaginal probiotic, to current recommended therapy for bacterial vaginosis (BV), oral metronidazole for 7 days, reduces 6 month recurrence rates

Methods Three arm randomised double-blind placebo controlled trial of 450 women (150 per arm): [MetPlac] oral metronidazole (7 days)/vaginal placebo (12 days), [MProb] oral metronidazole (7 days)/vaginal probiotic(12 days) and [MetClin] oral metronidazole ( 7 days)/vaginal clindamycin ( $1 \mathrm{~g} 2 \%$ nocte, 7 days). Symptomatic 18-50-year-old females with $\mathrm{BV}$ on vaginal swab by the Nugent method were enrolled at Melbourne Sexual Health Centre, Australia. Participants underwent initial examination \& STI screen and completed a detailed behavioural questionnaire at $0,1,2,3$ \& 6 months. At each interval participants were posted a kit containing swabs and a slide for self-collection and a questionnaire. Principle study outcome: Nugent score of 7-10. Cumulative BV recurrence rates were calculated and compared using $\chi^{2}$ and survival analyses using SPSS and STATA.

Results 450 women with BV were recruited from December 2007 to May 2010. Median age was 27 years (range 18-49), 210 (48\%) reported a past history of $\mathrm{BV}$; there were no significant differences in

Abstract 03-S5.05 Table 1

\begin{tabular}{|c|c|c|c|c|c|}
\hline Stage & $\mathbf{N}$ & $\begin{array}{l}\text { Median RPR } \\
\text { at diagnosis }\end{array}$ & $\begin{array}{l}\% \text { With increased titres } \\
\text { within } 14 \text { days following } \\
\text { treatment }(95 \% \mathrm{Cl})\end{array}$ & $\begin{array}{l}\% \text { With titres increased } \\
\text { by one dilution following } \\
\text { therapy ( } 95 \% \mathrm{CI})\end{array}$ & $\begin{array}{l}\% \text { With titres increased by } \\
\geq 2 \text { dilutions following } \\
\text { therapy }(95 \% \mathrm{Cl})\end{array}$ \\
\hline Primary & 115 & 1:16 & 30.4 (22.2 to 39.7$)$ & 80.0 (63.1 to 91.6$)$ & 20.0 (8.4 to 36.9$)$ \\
\hline Secondary & 218 & $1: 64$ & 17.0 (12.2 to 22.6$)$ & 97.3 (85.8 to 99.9 ) & $2.70(0.1$ to 14.2$)$ \\
\hline Early latent & 132 & $1: 32$ & $15.9(10.1$ to 23.3$)$ & 85.7 (63.7 to 97.0$)$ & $14.3(3.0$ to 36.3$)$ \\
\hline Total & 465 & $1: 64$ & 20.0 (16.5 to 23.9$)$ & 88.2 (79.8 to 93.9$)$ & 11.8 (6.1 to 20.2$)$ \\
\hline
\end{tabular}

